\title{
Gesundheitsökonomische Aspekte der Versorgung der Multiplen Sklerose
}

Anne Zimmermann, Tonio Schönfelder

\subsection{Datenlage -97}

5.2 Direkte Kosten - 98

5.2.1 Direkte medizinische Kosten - 99

5.2.2 Direkte nicht-medizinische Kosten - 101

5.3 Indirekte Kosten - 102

5.4 Intangible Kosten $\quad-104$

5.5 Krankheitslast -106

5.6 Finanzierung, Vergütung und Regularien der Arzneimittelversorgung -108

5.6.1 Morbiditätsorientierter Risikostrukturausgleich -108

5.6.2 Vergütung stationärer Leistungen - 108

5.6.3 Vergütung ambulanter Leistungen - 109

5.6.4 Regulatorische Aspekte der Arzneimittelversorgung - 109

Literatur -112 


\section{Zusammenfassung}

Die Versorgung von Patienten mit Multipler Sklerose ist mit hohen Gesundheitsausgaben verbunden. Die jährlichen direkten Durchschnittskosten liegen zwischen $21.932 €$ bis $23.087 €$ pro Patient. Diese variieren in Abhängigkeit von der Verlaufsform, dem Behinderungsgrad und der Schubaktivität. Mit einem Anteil von rund $60 \%$ bilden die Arzneimittel den größten Kostenblock innerhalb der direkten medizinischen Kosten, gefolgt von den Kosten für stationäre und ambulante Leistungen. Mit zunehmendem Behinderungsgrad gewinnen die direkten nichtmedizinischen Ausgaben an Bedeutung, insbesondere durch steigende Kosten für die informelle Pflege. Die jährlichen indirekten Kosten der Multiplen Sklerose variieren je nach Studie zwischen $16.911 €$ bis $19.384 €$ pro Patient. Der größte Anteil an den indirekten Kosten entsteht infolge von Erwerbsminderung bzw. Frühverrentung. Gemäß einer Analyse von Abrechnungsdaten gesetzlich Krankenversicherter bezogen im Jahr 2010 fast $50 \%$ der Patienten mit Multipler Sklerose eine Erwerbsminderungsrente im Vergleich zu rund $3 \%$ in der Gesamtbevölkerung. Mit zunehmender Schubaktivität steigen die Ausgaben an. Studien schätzen die direkten und indirekten Kosten eines Schubes auf $2.955 €$ bis $5.249 €$ pro Patient. Die Schubprophylaxe hat somit besondere Bedeutung in der Versorgung von Patienten mit Multipler Sklerose im Kontext begrenzter finanzieller Ressourcen. Die intangiblen Kosten werden auf durchschnittlich $10.000 €$ pro Patient geschätzt. Die größte Krankheitslast verursacht die Multiple Sklerose bei Patienten im Alter zwischen 30 bis 59 Jahren.

Im folgenden Kapitel werden gesundheitsökonomische Aspekte der Multiplen Sklerose (MS) dargestellt. Dazu zählt die Betrachtung der direkten, indirekten und intangiblen Kosten, soweit die vorhandene Daten- und Studienlage dies zulässt. Im Anschluss werden verschiedene Aspekte der Finanzierung und Vergütung beschrieben, u. a. auch die Nutzenbewertungsverfahren von MS-Arzneimitteln.

Direkte Kosten bezeichnen den Ressourcenverbrauch, der mit der Ausführung oder Anwendung einer Gesundheitsleistung verbunden ist (Graf von der Schulenburg u. Greiner 2007; IQWiG 2009). Dazu zählen beispielsweise Kosten für Arzneimittel, für diagnostische Leistungen wie die Liquoruntersuchung und die Durchführung einer Magnetresonanztomografie (MRT) sowie die Behandlung in einer Fachklinik für Patienten mit MS. Indirekte Krankheitskosten umfassen den mittelbar mit einer Krankheit verbundenen Ressourcenverlust (Statistisches Bundesamt 2010). Diese Kosten belasten Dritte in der Gesellschaft und somit nicht die Krankenkasse, weitere Kostenträger oder die Patienten selbst (Graf von der Schulenburg u. Greiner 2007). Sie entstehen durch Produktivitätsausfälle in Folge von Arbeits- und Erwerbsunfähigkeit oder vorzeitigen Tod (IQWiG 2009). Intangible Kosten in gesundheitsökonomischen Evaluationen stellen eine Bewertung von krankheitsbedingten Einschränkungen wie beispielsweise Schmerzen, Depressionen oder den Verlust an Lebensqualität dar (Gesundheitsberichterstattung des Bundes 2015a).

Zur Analyse der ökonomischen Belastung der MS stehen keine sektorenübergreifenden aggregierten Daten zur Verfügung. Die für diesen Zweck geeigneten Krankheitskostendaten des Statistischen Bundesamtes sind für MS nicht abrufbar. Daher basiert die folgende gesundheitsökonomische Betrachtung ausschließlich auf Kostenstudien. Bei der Auswertung dieser Studien muss berücksichtigt werden, dass deren Qualität sehr eng mit den einbezogenen Daten und der Studienmethodik zusammenhängt. Ein Vergleich zwischen den Studien ist oftmals schwierig, da u. a. unterschiedliche Zeitperioden und Kostenkomponenten betrachtet werden (Evers et al. 2004). Krankheitskostenstudien zur MS in Deutschland werden zu Beginn des folgenden Abschnitts beschrieben. Diese Kostenstudien werden für die Darstellung der direkten und indirekten Kosten herangezogen und ggf. mit weiteren Studien zu einzelnen Kostenkomponenten ergänzt.

In den Kostenstudien zur MS in Deutschland überwiegt die gesellschaftliche Perspektive. Diese gilt als die umfassendste Kostenbetrachtung, da sämtliche Kosten eingeschlossen werden, ohne Berücksichtigung des jeweiligen Kostenträgers (IQWiG 2009). Wenn die Datenlage es ermöglicht, werden in Einzelfällen zusätzlich die Kosten der gesetzlichen Krankenversicherung (GKV) abgebildet. 
- Tab. 5.1 Merkmale der Kostenstudien zur MS in Deutschland. (IGES - Hapfelmeier et al. (2013); Karampampa et al. (2012a); Karampampa et al. (2012b); Kobelt et al. (2006); Reese et al. (2011)

\begin{tabular}{|c|c|c|c|c|}
\hline Erstautor & Kobelt, 2006 & Karampampa, 2012 & Reese, 2011 & Hapfelmeier, 2013 \\
\hline Stichprobe (n) & 2.973 & 244 & 144 & 3.007 \\
\hline $\begin{array}{l}\text { Jahr der Kosten- } \\
\text { schätzung }\end{array}$ & 2005 & 2009 & 2009 & 2009-2010 \\
\hline Datenerhebung & Befragung & Befragung & Befragung & Routinedaten \\
\hline Rekrutierung & $\begin{array}{l}3 \text { Kliniken, } 3 \text { Privatpraxen, } \\
\text { Patientendatenbank }\end{array}$ & $\begin{array}{l}\text { MS-Behandlungs- } \\
\text { zentren }\end{array}$ & MS-Ambulanz & - \\
\hline Alter in Jahren $(\varnothing)$ & $45,1 \pm 11,1$ & $41,2 \pm 9,9$ & $41,7 \pm 11,3$ & k. A. \\
\hline EDSS-Wert ( $\varnothing)$ & $3,8 \pm 2,3$ & $1,8 \pm 1,8$ & $3,5 \pm 2,0$ & k. A. \\
\hline MS-Verlaufsform & $\begin{array}{l}\text { RRMS: } 40 \% \\
\text { SPMS+PPMS: } 47 \% \\
\text { NO: } 13 \%\end{array}$ & $\begin{array}{l}\text { RRMS: } 65 \% \\
\text { SPMS: } 13 \% \\
\text { PPMS: } 8 \% \\
\text { NO: } 14 \%\end{array}$ & $\begin{array}{l}\text { RRMS: } 67 \% \\
\text { SPMS: } 28 \% \\
\text { PPMS: } 5 \%\end{array}$ & k. A. \\
\hline
\end{tabular}

k. A.: keine Angabe, RRMS: schubförmig-remittierende MS; SPMS: sekundär-progrediente MS, PPMS: primär-progrediente MS; NO: Verlaufsform unbekannt oder nicht angegeben, Ø: Durchschnitt

Anmerkungen: Zum Vergleich die Merkmale des DMSG-Datensatzes 2014: Durchschnittsalter: 45,6 Jahre; durchschnittlicher EDSS-Wert: 3,5; MS-Verlaufsformen: RRMS $59 \%$; SPMS $26 \%$, PPMS 8 \%, KIS 4 \%, Unbekannte Verlaufsform $4 \%$ (msfp 2014)

\subsection{Datenlage}

Mittels einer strukturierten Literaturrecherche konnten insgesamt vier Krankheitskostenstudien identifiziert werden, die Daten zu den Kosten der MS in Deutschland liefern. Studien, die Daten vor dem Jahr 2000 erhoben haben, wurden aufgrund ihrer fehlenden Aktualität ausgeschlossen. Eine Übersicht der Studien und ihrer Merkmale ist in - Tab. 5.1 dargestellt.

Die internationale Krankheitskostenstudie von Kobelt et al. (2006) hat Daten von 2.973 Patienten mit MS in Deutschland erhoben. Die Patienten wurden über drei Kliniken, drei auf MS spezialisierten Privatpraxen sowie über eine Patientendatenbank rekrutiert und schriftlich befragt. Die Kostenberechnung aus gesellschaftlicher Perspektive beinhaltet direkte und indirekte Kosten für das Jahr 2005.

Die internationale Studie TRIBUNE (Treatment Experience, Burden and Unmet Needs of MS) liefert die aktuellsten Daten zu den Kosten der MS (Karampampa et al. 2012a). In Deutschland wurden dafür 244 Patienten über MS-Behandlungszentren rekrutiert und schriftlich befragt (Karampampa et al. 2012b). Die genaue Anzahl und Lage der Behandlungszentren ist nicht angegeben. Die Studie schätzt die direkten und indirekten gesellschaftlichen Kosten der MS für das Jahr 2009 in Abhängigkeit der Verlaufsform und des Behinderungsgrads. Eine Darstellung der Gesamtkosten für einen durchschnittlichen Patienten mit MS erfolgt nicht.

Für die Kostenstudie von Reese et al. (2011) wurden in den Jahren 2007 und 2008144 Patienten in der MS-Ambulanz des Universitätsklinikums Marburg rekrutiert. Die Datenerhebung erfolgte durch eine Befragung der Patienten. Die Studie schätzt die gesellschaftlichen direkten und indirekten Kosten für das Jahr 2009. Die Kostenerhebung erfolgte über einen Zeitraum von drei Monaten. Daher wurden die Angaben zur Vergleichbarkeit mit den anderen Kostenstudien auf ein Jahr hochgerechnet.

Bei der Studie von Hapfelmeier et al. (2013) handelt es sich um eine Analyse von Abrechnungsdaten der GKV. Die Studie ermittelt die direkten 
Kosten für Arzneimittel, Arzt- und Krankenhausbehandlungen in Abhängigkeit des eingesetzten immunmodulatorischen Arzneimittels für die Jahre 2009 und 2010. Für die Analyse standen Daten von ca. 1 Mio. Versicherten zur Verfügung. Die Ergebnisse basieren auf einer repräsentativen Stichprobe von 3.007 durchgehend Versicherten mit einer gesicherten MS-Diagnose (ICD-10-Code: G35). Patienten mit einer gesicherten MS-Diagnose werden in der Studie definiert als Patienten mit mindestens zwei MS-Diagnosen oder mindestens einer MSDiagnose und zusätzlich einer Verordnung eines immunmodulatorisch wirksamen Arzneimittels zur MS-Therapie (Hapfelmeier et al. 2013). Die Betrachtungsperspektive ist in diesem Fall die GKV (- Tab. 5.1).

8 Ein direkter Vergleich der Kosten zwischen den Studien ist nur eingeschränkt möglich, da sich die Studien u. a. hinsichtlich der Abdeckung und Aktualität der Daten sowie der Zusammensetzung der Studienpopulation unterscheiden bzw. relevante Angaben zur Interpretation der Daten fehlen.

Kobelt et al. (2006) liefern flächendeckende Daten für die MS-Population in Deutschland für das Jahr 2005, die im Hinblick auf Alter und EDSS-Wert ( $\triangleright$ Kap. 3) ähnlich zum DMSG-Datensatz (msfp 2014) sind. Bei der Studie von Karampampa et al. (2012) ist unklar, wie viele und welche Behandlungszentren in die Studie involviert wurden. Es fällt allerdings auf, dass die eingeschlossenen $\mathrm{Pa}$ tienten jünger sind und einen geringeren Behinderungsgrad aufweisen im Vergleich zu den anderen Studien und zum DMSG-Datensatz (msfp 2014). Die Ergebnisse von Reese et al. (2011) basieren auf nur einer MS-Ambulanz und sind somit nur eingeschränkt auf die Grundgesamtheit der Patienten mit MS in Deutschland übertragbar. Hapfelmeier et al. (2013) liefern flächendeckende Abrechnungsdaten für die MS-Population in Deutschland, allerdings sind aufgrund fehlender Angaben zur Patientenpopulation (Alter, Behinderungsgrad, Verlaufsform) keine Aussagen zur Repräsentativität der Studienpopulation möglich. Darüber hinaus unterscheiden sich die Studien in den berücksichtigten Kostenarten und ihrer Berechnung. So werden beispielsweise in der Studie von Reese et al. (2011) keine Angaben zu den Kosten von diagnostischen Verfahren oder ambulanter Pflege gemacht. In der Regel werden in den Studien die zur Berechnung genutzten Datenquellen angegeben, allerdings fehlen oftmals detaillierte Angaben, um die Rechenschritte nachvollziehen zu können. Insbesondere bei den Kosten der informellen Pflege sowie den indirekten Kosten zeigen sich zwischen den Studien Unterschiede in der Vorgehensweise der Kostenberechnung.

\subsection{Direkte Kosten}

Die direkten Kosten der Behandlung setzen sich zusammen aus direkten medizinischen Kosten und direkten nicht-medizinischen Kosten. Direkte medizinische Kosten entstehen unmittelbar durch den Ressourcenverbrauch im Zusammenhang mit der Gesundheitsversorgung (Graf von der Schulenburg u. Greiner 2007; IQWiG 2009). Dazu zählen Krankenhausaufenthalte, ambulante Arztbesuche, diagnostische Leistungen und Medikamente. Als direkte nicht-medizinische Kosten werden Ausgaben bezeichnet, die unterstützend zur Erstellung der medizinischen Leistung wirken und in Folge der Erkrankung oder Behandlung entstehen. Dazu gehören beispielsweise Transportkosten, Kosten für Anschaffungen und Umbauten, Kosten für eine Haushaltshilfe sowie der bewertete Zeitaufwand von Patienten und pflegenden Angehörigen (Graf von der Schulenburg u. Greiner 2007; IQWiG 2009). Eine Übersicht über die direkten medizinischen und nicht-medizinischen Kosten der MS ist in - Tab. 5.2 dargestellt.

Die direkten durchschnittlichen Kosten für einen Patienten mit MS liegen je nach betrachteter Studie zwischen $21.932 €$ bis $23.087 €$ (Kobelt et al. 2006; Reese et al. 2011). Sie variieren jedoch in Abhängigkeit von der Verlaufsform, dem Behinderungsgrad und der Schubaktivität (Karampampa et al. 2012b; Kobelt et al. 2006; Reese et al. 2011). Werden nur die Kosten der GKV betrachtet, d. h. Ausgaben, die den Patienten oder Dritten entstehen, bleiben unberücksichtigt, liegt die Kostenspanne zwischen $15.583 €$ und $21.376 €$ pro Patient (Kobelt et al. 2006; Reese et al. 2011). 
- Tab. 5.2 Vergleich der jährlichen direkten Kosten der MS in € pro Patient (IGES - Hapfelmeier et al. (2013); Karampampa et al. (2012b); Kobelt et al. (2006); Reese et al. (2011)

\begin{tabular}{|c|c|c|c|c|}
\hline \multirow[b]{2}{*}{ Erstautor und Jahr der Kostenschätzung } & \multicolumn{3}{|c|}{ Gesellschaftliche Perspektive } & \multirow{2}{*}{$\begin{array}{l}\text { GKV-Perspektive } \\
\text { Hapfelmeier }{ }^{4} 2009\end{array}$} \\
\hline & $\begin{array}{l}\text { Kobelt } \\
2005\end{array}$ & $\begin{array}{l}\text { Karampampa } \\
2009\end{array}$ & $\begin{array}{l}\text { Reese } \\
2006\end{array}$ & \\
\hline \multicolumn{5}{|l|}{ Kostenarten } \\
\hline $\begin{array}{l}\text { Stationäre Behandlung, inkl. Rehabilitation } \\
\text { und Pflege }\end{array}$ & 3.203 & $1.087-3.162$ & 4.232 & $1.357-2.578^{9}$ \\
\hline $\begin{array}{l}\text { Ambulante Behandlung, inkl. } \\
\text { Heilmittelbehandlungen }\end{array}$ & 2.193 & $655-1.246$ & 1.936 & $770-1.459^{10}$ \\
\hline Ambulante Pflege & 903 & $114-1.707$ & k. A. & k. A. \\
\hline Diagnostik & 368 & $78-116$ & k. A. & k. A. \\
\hline Medikamente & 10.498 & $10.783-16.450$ & 14.880 & 6.767 \\
\hline Direkte medizinische Kosten (Summe) & 17.165 & $14.788-21.756$ & k. A. & k. A. \\
\hline Anschaffungen und Umbauten ${ }^{5}$ & 989 & $622-2.208$ & $768^{7}$ & k. A. \\
\hline Transport & 80 & k. A. & k. A. & k. A. \\
\hline Professionelle Unterstützung ${ }^{6}$ & 447 & $21-491$ & k. A. & k. A. \\
\hline Informelle Pflege & 4.407 & $1.482-12.919$ & k. A. & k. A. \\
\hline Direkte nicht-medizinische Kosten (Summe) & 5.922 & $2.612-14.887$ & k. A. & k. A. \\
\hline Direkte Kosten (Summe) & 23.087 & $17.400-35.280$ & $21.932^{8}$ & k. A. \\
\hline $\begin{array}{l}{ }^{1} \text { Bei der gesellschaftlichen Perspektive werde } \\
\text { ligen Kostenträgers (einschließlich der Kosten } \\
{ }^{2} \text { Bei der GKV-Perspektive werden nur Kosten, } \\
{ }^{3} \text { Die Kostenspanne ergibt sich in Abhängigke } \\
{ }^{4} \text { Die Kostenspanne ergibt sich in Abhängigke } \\
{ }^{5} \text { Beinhaltet Kosten für Hilfsmittel wie z. B. Gel } \\
{ }^{6} \text { Beinhaltet Kosten für eine Haushaltshilfe od } \\
{ }^{7} \text { In der Studie von Reese et al. (2012) wird die } \\
{ }^{8} \text { Zuzahlungen (Copayments) von } 140 € \text { werde } \\
{ }^{9} \text { Kosten für die stationäre Rehabilitation und } \\
{ }^{10} \text { Kosten für Heilmittelbehandlungen wurder }\end{array}$ & $\begin{array}{l}\text { sämtlich } \\
\text { ür Patien } \\
\text { lie der ge } \\
\text { der MS-- } \\
\text { vom ein } \\
\text { Ifen und } \\
\text { ambular } \\
\text { er Kosten } \\
\text { nicht se } \\
\text { flege wu } \\
\text { icht ber }\end{array}$ & $\begin{array}{l}\text { ten eingeschloss } \\
\text { chen Krankenkas } \\
\text { ffsform. } \\
\text { zten immunmod } \\
\text { ühle sowie Anpa } \\
\text { ge Krankenschw } \\
\text { den direkten Ko } \\
\text { aufgeführt, sind j } \\
\text { hicht berücksicht } \\
\text { chtigt. }\end{array}$ & $\begin{array}{l}\text { hne Berüc } \\
\text { ttstehen, b } \\
\text { gen zchen Ar } \\
\text { zugeordne } \\
\text { h in den di }\end{array}$ & $\begin{array}{l}\text { htigung des jewei- } \\
\text { cksichtigt. } \\
\text { imittel. } \\
\text { to oder Haus. } \\
\text { en Kosten enthalten. }\end{array}$ \\
\hline
\end{tabular}

\subsubsection{Direkte medizinische Kosten}

Kobelt et al. (2006) geben die direkten medizinischen Kosten der MS mit durchschnittlich 17.165€ pro Patient an. Die Kosten variieren jedoch in Abhängigkeit der Verlaufsform, wobei diesbezügliche Studienergebnisse nicht konsistent sind (Karampampa et al. 2012b; Reese et al. 2011). In der Studie von Karampampa et al. (2012) verursachen Patienten mit sekundär-progredienter MS (SPMS) aufgrund der höheren Ausgaben für Arzneimittel und stationärer sowie ambulanter Behandlung mit rund $21.800 €$ die höchsten direkten medizinischen Kosten im Vergleich zu den anderen Verlaufsformen. Für schubförmig-remittierende MS (RRMS) werden Kosten von rund 17.800 Euro angegeben und für primär-progrediente (PPMS) rund $16.800 €$ (Karampampa et al. 2012b). In der Studie von Reese et al. (2011) sind stattdessen die Kosten (exkl. Kosten für Pflege und diagnostische Verfahren) für Patienten mit RRMS am höchsten, möglicherweise aufgrund des hohen Anteils von Patienten mit Im- 


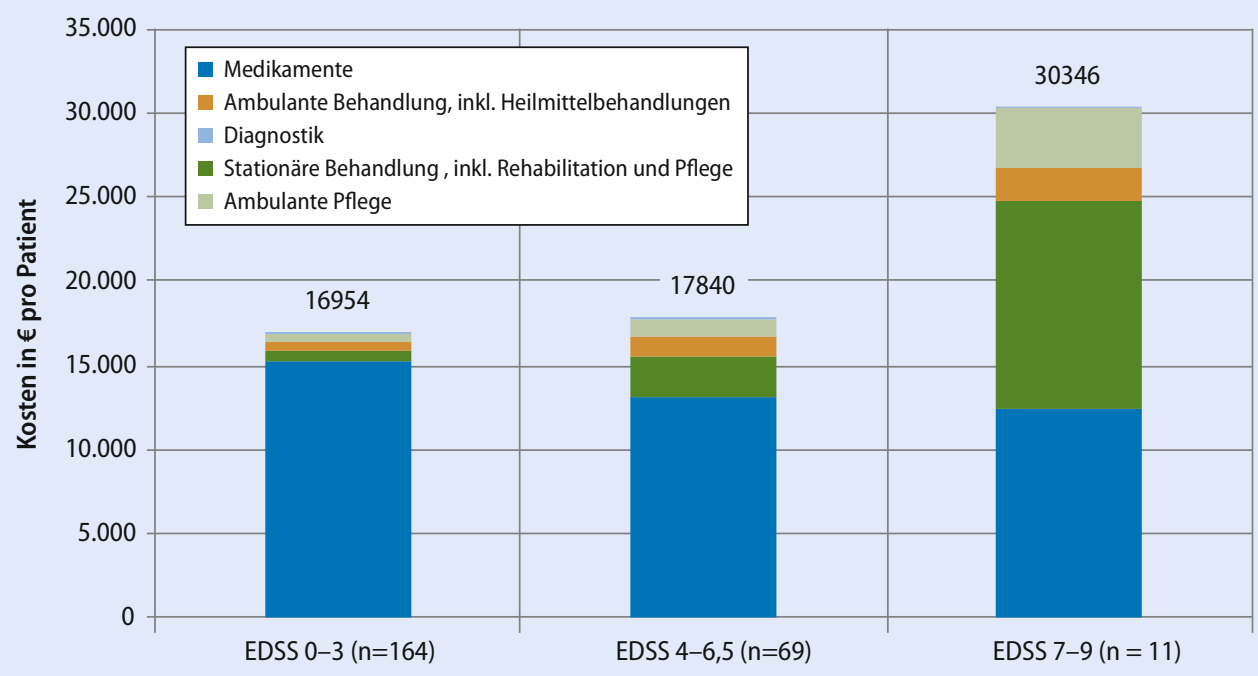

- Abb. 5.1 Jährliche direkte medizinische Kosten der MS nach Behinderungsgrad in 2009 Quelle: IGES - Karampampa et al. (2012b)

muntherapie (89\%) und den damit verbundenen hohen Arzneimittelkosten. Patienten mit PPMS verursachen auch in dieser Studie die geringsten direkten medizinischen Kosten (Reese et al. 2011).

Ausgelöst durch einen höheren Behinderungsgrad steigen die direkten medizinischen Kosten von Patienten mit MS aufgrund zunehmender stationärer und pflegerischer Leistungen an (• Abb. 5.1).

Darüber hinaus verursachen Patienten mit Schüben höhere direkte medizinische Kosten als Patienten ohne Schubaktivität, insbesondere durch höhere Ausgaben für stationäre und ambulante Behandlungen (Karampampa et al. 2012b; Kobelt et al. 2006). Kobelt et al. geben die Gesamtkosten eines Schubes für Patienten mit einem EDSS-Wert $<5$ mit $2.955 €$ an (Kobelt et al. 2006). Karampampa et al. schätzen den durchschnittlichen Kostenunterschied von Patienten mit RRMS, einem EDSS-Wert $\leq 5$ und einem oder mehreren Schüben innerhalb eines Jahres im Vergleich zu Patienten ohne Schübe auf $5.249 €$ (Karampampa et al. 2012b). Beide Kostenangaben beinhalten neben den direkten Kosten auch die indirekten Kosten eines Schubes $(\triangleright A b$ schn. 5.3). Eine Aufteilung nach Kostenarten lassen die Studiendaten nicht zu.

In allen einbezogenen Studien bilden die Ausgaben für Arzneimittel mit $10.498 €$ bis $14.880 €$ den größten Kostenblock innerhalb der direkten medizinischen Kosten (Karampampa et al. 2012b; Kobelt et al. 2006; Reese et al. 2011). Eine Ausnahme bildet die Studie von Hapfelmeier et al. (2013) basierend auf der Analyse von Routinedaten der GKV. Die durchschnittlichen Arzneimittelkosten für alle $\mathrm{Pa}$ tienten mit MS betrugen im Jahr $20096.767 €$ und im Jahr $20107.083 €$ pro Patient (Hapfelmeier et al. 2013). Im Vergleich zu den übrigen Studien sind diese Kosten wesentlich niedriger, was vor allem auf einen deutlich geringeren Anteil von Patienten mit Immuntherapie zurückzuführen ist. In den Abrechnungsdaten der GKV liegt der Anteil der Patienten mit MS-Therapeutika bei 41,5\%, während ihr Anteil in den anderen Studien bei $50 \%-80$ \% liegt. Darüber hinaus berücksichtigen die GKV-Daten keine Ausgaben der Patienten für freiverkäufliche Arzneimittel oder Medikamentenzuzahlungen. Arzneimittel, die in den letzten Jahren eingeführt worden sind, wie Fingolimod (Erstzulassung EU 2011), Fampridin (Erstzulassung EU 2011), Teriflunomid (Erstzulassung EU 2013), Alemtuzumab (Erstzulassung EU 2013) und Dimethylfumarat (Erstzulassung EU 2014) ( Abschn. 5.6.4), sind in diesen Kostenberechnungen noch nicht berücksichtigt.

Die Arzneimittelkosten sind - vermutlich aufgrund fehlender Therapieoptionen - bei Patienten 
mit PPMS am niedrigsten (Karampampa et al. 2012b; Reese et al. 2011). Mit zunehmendem Behinderungsgrad sinken sie, da die Immuntherapien insbesondere bei Patienten im frühen Krankheitsverlauf mit geringerem Behinderungsgrad eingesetzt werden (siehe - Abb. 5.1) (Karampampa et al. 2012b; Reese et al. 2011).

Mit einem Anteil von $88 \%$ verursachen die immunmodulatorischen Arzneimittel (IMA) die meisten Medikamentenkosten. Die durchschnittlichen Kosten für Patienten, die mit einem IMA behandelt werden, lagen bei $14.929 €$ in 2009 bzw. $15.780 €$ pro Patient in 2010 (Hapfelmeier et al. 2013). Die Arzneimittelkosten variieren zwischen den IMA stark. Die geringsten jährlichen Kosten verursachen Patienten, die mit Azathioprin behandelt werden (450 € pro Patient). Die höchsten Kosten verursachen Patienten, die mit Natalizumab (25.104 € pro Patient) behandelt werden. Etwa $11 \%$ der Arzneimittelkosten werden durch sonstige Arzneimittel, insbesondere durch Kortikoide zur Schubbehandlung und Hilfsmittelverordnungen verursacht. Die Kosten für die medikamentöse Symptombehandlung der MS sind mit $1 \%$ gering. Die durchschnittlichen jährlichen Kosten für Patienten, die symptomatisch behandelt werden, lagen bei $272 €$ in 2009 und $256 €$ in 2010 pro Patient (Hapfelmeier et al. 2013). Patienten mit MS geben durchschnittlich zwischen $81 €$ und $290 €$ für freiverkäufliche Präparate aus (Karampampa et al. 2012b; Kobelt et al. 2006).

Den zweitgrößten Kostenblock bei den direkten medizinischen Kosten bilden die Ausgaben im stationären Bereich. Die stationären Kosten werden je nach Studie auf $3.203 €$ bis $4.232 €$ pro Patient geschätzt (Kobelt et al. 2006; Reese et al. 2011). Die Kostenunterschiede zwischen den Studien sind aufgrund fehlender Angaben schwer nachzuvollziehen, werden aber möglicherweise insbesondere durch unterschiedliche Vorgehensweisen bei der Kostenschätzung herbeigeführt. Der größte Teil der Kosten wird durch die stationäre Behandlung im Krankenhaus verursacht, hauptsächlich in den neurologischen Abteilungen (Kobelt et al. 2006). Zwischen $23 \%$ bis $32 \%$ der Kosten entstehen im Bereich der stationären Rehabilitation (Kobelt et al. 2006; Reese et al. 2011). Bei den Kosten für stationäre Behandlungen zeigt sich eine große Abhängig- keit vom Behinderungsgrad der Patienten mit MS; auf Patienten mit höheren Werten auf der EDSS entfallen signifikant höhere Kosten (• Abb. 5.1). Darüber hinaus verursachen Patienten mit primärprogredienter Verlaufsform (3.162€) und sekundär-progredienter Verlaufsform (2.316 $€$ ) höhere stationäre Kosten als Patienten mit schubförmigem Verlauf (1.455 €) (Karampampa et al. 2012b).

Die durchschnittlichen ambulanten Behandlungskosten liegen je nach betrachteter Studie zwischen $1.936 €$ und $2.193 €$ pro Patient (Kobelt et al. 2006; Reese et al. 2011). Die Kosten variieren in Abhängigkeit von der Verlaufsform der MS: Bei Patienten mit unbekannter Verlaufsform oder RRMS sind die ambulanten Behandlungskosten am geringsten. Die ambulanten Kosten steigen in Abhängigkeit vom Ausmaß des Behinderungsgrades (• Abb. 5.1). Mehr als die Hälfte der Kosten im ambulanten Bereich wird durch Heilmittelbehandlungen wie Physio- oder Ergotherapien verursacht (Kobelt et al. 2006; Reese et al. 2011). Weitere direkte medizinische Kosten entstehen durch Ausgaben für die ambulante Pflege. In der Studie von Kobelt et al. (2006) liegen die ambulanten Pflegekosten bei durchschnittlich $903 €$ pro Patient. Die Pflegekosten sind für Patienten mit SPMS (491€) und PPMS (178 €) deutlich höher als bei Patienten mit RRMS $(141 €)$ oder unbekannter Verlaufsform $(21 €)(\mathrm{Ka}-$ rampampa et al. 2012b). Sie steigen aufgrund des erhöhten Pflegeaufwands mit zunehmender Behinderung an. Einen geringen Anteil an den direkten medizinischen Kosten haben diagnostische Leistungen. Bei Kobelt et al. (2006) liegen diese Kosten bei $368 €$ pro Patient. Etwa $80 \%$ davon werden durch Untersuchungen des Kopfes und der Wirbelsäule mittels MRT verursacht, rund $4 \%$ durch die Aufzeichnung evozierter Potenziale und weniger als $1 \%$ der Kosten für diagnostische Leistungen entfallen auf die Liquoruntersuchung (Kobelt et al. 2006).

\subsubsection{Direkte nicht-medizinische Kosten}

Den größten Kostenfaktor innerhalb der direkten nicht-medizinischen Kosten stellt die informelle Pflege dar (•Tab. 5.2). Zur Ermittlung wird die Zeit erfasst, die Angehörige oder Freunde mit der Betreuung des an MS Erkrankten verbringen, und die- 


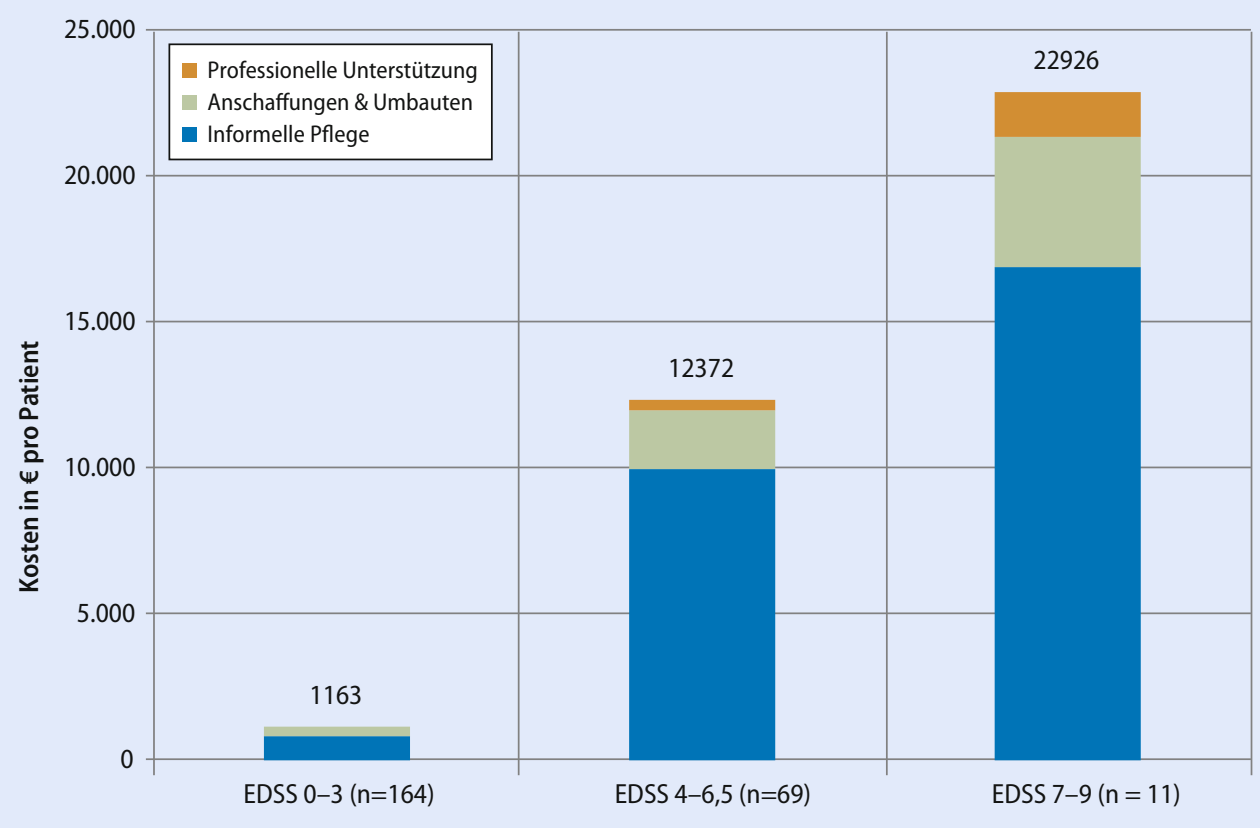

- Abb. 5.2 Direkte nicht-medizinische Kosten der MS in 2009 Quelle: IGES - Karampampa et al. (2012b)

se wird mit dem entgangenen Einkommen der Betreuenden bewertet. In der Studie von Kobelt et al. (2006) nehmen 47,5\% der Patienten durchschnittlich 87 Stunden pro Monat pflegerische Leistungen durch Angehörige in Anspruch (Kobelt et al. 2006). Die jährlichen Kosten für die informelle Pflege werden auf durchschnittlich $4.407 €$ pro Patient geschätzt, sind jedoch stark abhängig vom Grad der Behinderung und konzentrieren sich überwiegend auf die höheren EDSS-Stufen (Karampampa et al. 2012b) (• Abb. 5.2).

Die Kosten für Anschaffungen und Umbauten liegen je nach Studie zwischen $768 €$ und $989 €$ pro Patient (Kobelt et al. 2006, Reese et al. 2011). Sie steigen mit zunehmender Behinderung stark an (• Abb. 5.2).

Weitere direkte nicht-medizinische Kosten entstehen durch die professionelle Unterstützung beispielsweise durch eine Haushaltshilfe oder eine ambulant tätige Krankenschwester. Kobelt et al. (2006) schätzen die Kosten dafür auf $447 €$ pro Patient. Diese Leistungen werden insbesondere von Patienten mit höherer EDSS-Stufe in Anspruch genom- men (• Abb. 5.2). Transportkosten bilden mit ca. $80 €$ einen geringen Anteil der direkten nicht-medizinischen Kosten (Kobelt et al. 2006).

\subsection{Indirekte Kosten}

Die Mehrheit der an MS-Erkrankten erhält die Diagnose zwischen dem 20. und 40. Lebensjahr. Somit sind insbesondere jüngere Menschen in ihrer produktiven Lebensphase von der Erkrankung betroffen. Es ist daher davon auszugehen, dass den indirekten krankheitsassoziierten Kosten infolge von Produktivitätsausfällen durch Arbeits- und Erwerbsunfähigkeit oder vorzeitigem Tod eine hohe Relevanz zukommt.

Gemäß den Daten des DMSG-Datensatzes aus dem Jahr 2005/2006 sind etwa 37 \% der Patienten berufstätig, davon $76 \%$ in Vollzeit (Flachenecker et al. 2008). Knapp $40 \%$ der Patienten beziehen nach einer durchschnittlichen Krankheitsdauer von 13 Jahren (Durchschnittsalter 44 Jahre) Rentenleistungen aufgrund von krankheitsbedingten Einschrän- 


\begin{tabular}{|c|c|c|c|}
\hline Erstautor und Jahr der Kostenschätzung & $\begin{array}{l}\text { Kobelt } \\
2005\end{array}$ & $\begin{array}{l}\text { Karampampa }{ }^{1} \\
2009\end{array}$ & $\begin{array}{l}\text { Reese } \\
2006\end{array}$ \\
\hline Arbeitsunfähigkeit (kurz- und langfristig) & 2.137 & $101-1.884$ & 5.188 \\
\hline $\begin{array}{l}\text { Teilweise oder vollständige Erwerbsminderung/ } \\
\text { Frühberentung }\end{array}$ & 14.774 & $2.573-8.085$ & 13.088 \\
\hline Arbeitslosigkeit & k. A. & k. A. & 524 \\
\hline Reduzierung der Wochenarbeitszeit & k. A. & k. A. & 584 \\
\hline Indirekte Kosten gesamt & 16.911 & $4.457-9.702$ & 19.384 \\
\hline
\end{tabular}

kungen bei der Arbeit (Flachenecker et al. 2008). Der Anteil der Patienten mit Erwerbsminderung steigt in Abhängigkeit vom Alter und dem Behinderungsgrad der Patienten. Bereits $15 \%$ der Patienten mit einem EDSS-Wert von $\leq 3,5$ beziehen Rentenleistungen, obwohl diese Patienten noch gehfähig sind. Dies lässt die Schlussfolgerung zu, dass neben den physischen Defiziten auch andere Beschwerden eine Rolle spielen. Probleme in der Mobilität und Motorik gelten als sichere Prädiktoren für eine vorzeitige Berentung (Kern et al. 2013). Darüber hinaus können aber auch andere Symptome den Arbeitsalltag für die Patienten erschweren. Fatigue, Angst, Depressionen, Schmerzen und kognitive Defizite können zu einer eingeschränkten Konzentrations- und Gedächtnisfähigkeit führen. Störungen beim Schlucken und Sprechen erschweren die Kommunikation, und visuelle Tätigkeiten können bei Sehstörungen nur eingeschränkt durchgeführt werden.

Eine Analyse der Abrechnungsdaten von Versicherten der BARMER GEK für das Jahr 2010 kommt zu dem Ergebnis, dass beinahe die Hälfte der Patienten mit MS fast durchgängig eine Erwerbsminderungsrente bezog. In der Gesamtbevölkerung lag dieser Anteil bei 2,9\% (IGES Institut 2014). Die Studie von Kobelt et al. (2006) berichtet ähnliche Daten: der Anteil der Patienten im vorzeitigen Ruhestand infolge der MS lag bei $34 \%$. Die Patienten waren im Durchschnitt 40 Jahre alt, als sie vorzeitig berentet wurden (Kobelt et al. 2006).

Die Therapien in der Behandlung von Patienten mit MS haben auch das Ziel, eine aktive Teilnahme des Patienten am Arbeitsleben zu ermöglichen. Versorgungsökonomische Betrachtungen zum langfristigen Nutzen der verschiedenen Therapieansätze sind aktuell nicht verfügbar.

Die sich aus den Produktivitätsverlusten ergebenden indirekten Kosten der MS wurden in verschiedenen Kostenstudien geschätzt. Eine Übersicht der indirekten Kosten für die ausgewählten Studien ist in - Tab. 5.3 dargestellt.

Je nach Studie variieren die durchschnittlichen indirekten Kosten für einen Patienten mit MS zwischen $16.911 €$ und $19.384 €$ (•Tab. 5.3) (Kobelt et al. 2006; Reese et al. 2011). In der Studie von Karampampa et al. (2012b) liegen die indirekten Kosten deutlich niedriger im Vergleich zu den anderen Studien. Ursache dafür ist möglicherweise die jüngere Studienpopulation mit einem geringeren Behinderungsgrad, die infolgedessen auch einen geringeren Anteil von Erkrankten in vorzeitigem Ruhestand aufweist (9\% im Vergleich zu $34 \%$ ). Darüber hinaus kommt es zu Abweichungen zwischen den Studien aufgrund unterschiedlicher Ansätze bei der Kostenberechnung.

Den größten Kostenblock innerhalb der indirekten Kosten bilden die Kosten für Rentenleistungen infolge einer teilweisen oder vollständigen Erwerbsminderung. Je nach Studie variieren die Kosten zwischen $13.088 €$ und $14.774 €$ pro Patient (Kobelt et al. 2006; Reese et al. 2011). Weitere indirekte Kosten entstehen durch Arbeitsunfähigkeit, Arbeitslosigkeit oder durch die Reduzierung der Arbeitszeit. Die indirekten Kosten unterscheiden 


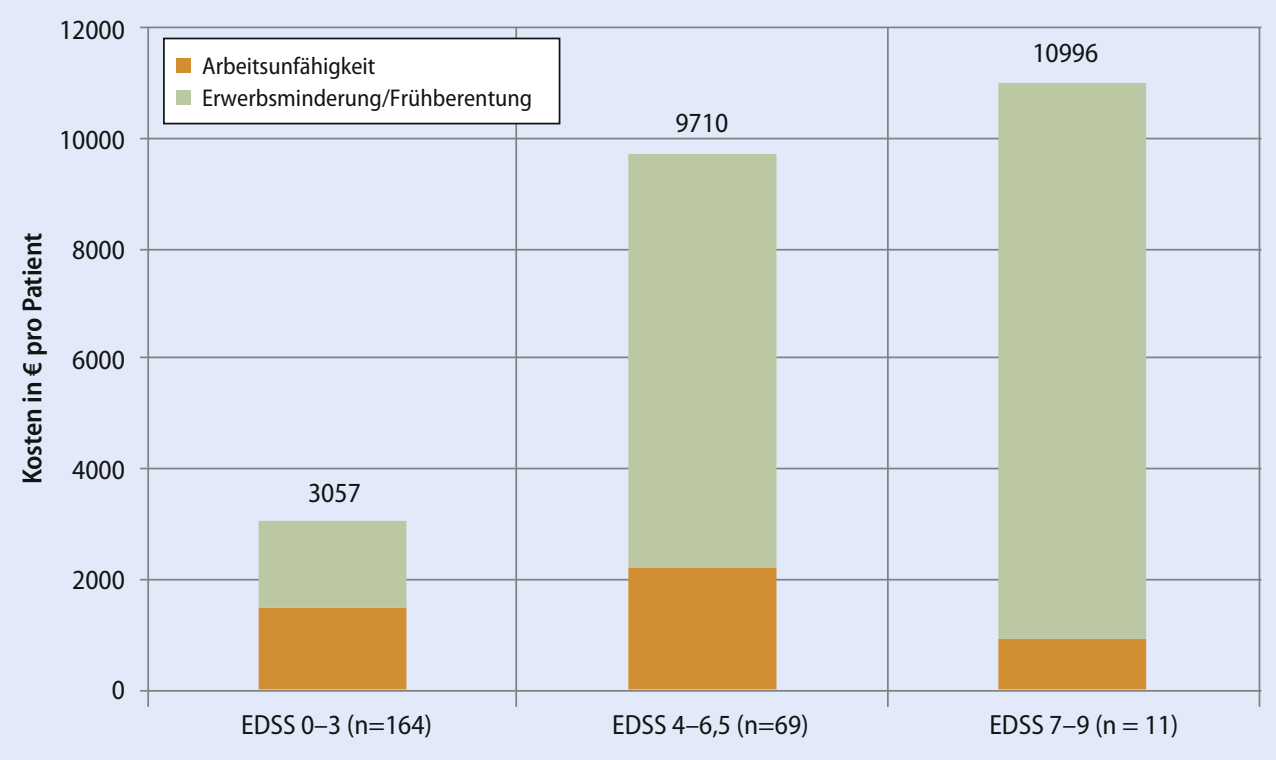

- Abb. 5.3 Indirekte Kosten der MS nach EDSS-Stufe in €, Jahr 2009

Quelle: IGES - Karampampa et al. (2012b)

sich in Abhängigkeit von der Verlaufsform. Patienten mit progredienter Verlaufsform verursachen höhere indirekte Kosten als Patienten mit RRMS, ausgelöst vor allem durch höhere Kosten infolge der Erwerbsminderung (Karampampa et al. 2012b; Reese et al. 2011). Darüber hinaus steigen die indirekten Kosten in Abhängigkeit vom Behinderungs$\operatorname{grad}(\bullet$ Abb. 5.3).

Die indirekten Kosten sind auch für Patienten mit Schüben höher als bei Patienten ohne Schubaktivität, insbesondere durch höhere Kosten infolge von Arbeitsunfähigkeit (Kobelt et al. 2006).

Ein weiterer Indikator für die indirekten krankheitsassoziierten Kosten ist die vorzeitige Mortalität auf Basis der potenziell verlorenen Lebensjahre (Potential Years of Life Lost) durch eine Krankheit (Gesundheitsberichterstattung des Bundes 2015a; Graf von der Schulenburg u. Greiner 2007). Dabei wird auch die nicht-erwerbstätige Bevölkerung in die ökonomische Betrachtung mit einbezogen (Löwel et al. 2006). Errechnet werden die verlorenen Lebensjahre als Differenz aus dem tatsächlichen Sterbealter und dem 70. Lebensjahr (Gesundheitsberichterstattung des Bundes 2015b). Es wird angenommen, dass die Gestorbenen ohne die Erkran- kung 70 Jahre alt geworden wären. Die Daten werden vom Statistischen Bundesamt zur Verfügung gestellt.

Im Jahr 2013 sind in Deutschland 773 Personen (278 Männer und 495 Frauen) vor ihrem 70. Lebensjahr an MS verstorben. Die errechnete Zahl der verlorenen Lebensjahre in 2013 liegt für beide Geschlechter bei 10.198 Jahren. Mit $62 \%$ entfällt die Mehrheit der verlorenen Lebensjahre auf Frauen. Die Anzahl der vorzeitig Gestorbenen infolge der MS hat sich seit 2003 mit 740 kaum verändert. Daher ist auch bei der Zahl der verlorenen Lebensjahre infolge der MS über die letzten zehn Jahre weder ein wesentlicher An- noch Abstieg zu erkennen (• Abb. 5.4).

\subsection{Intangible Kosten}

Intangible Kosten bezeichnen monetär nicht messbare Effekte infolge der Erkrankung wie z. B. Einschränkungen durch Schmerzen, Depressionen oder den krankheitsbedingten Verlust der Lebensqualität (IQWiG 2009). Eine Querschnittsstudie mit 3.157 Mitgliedern der Deutschen Multiple Sklerose 


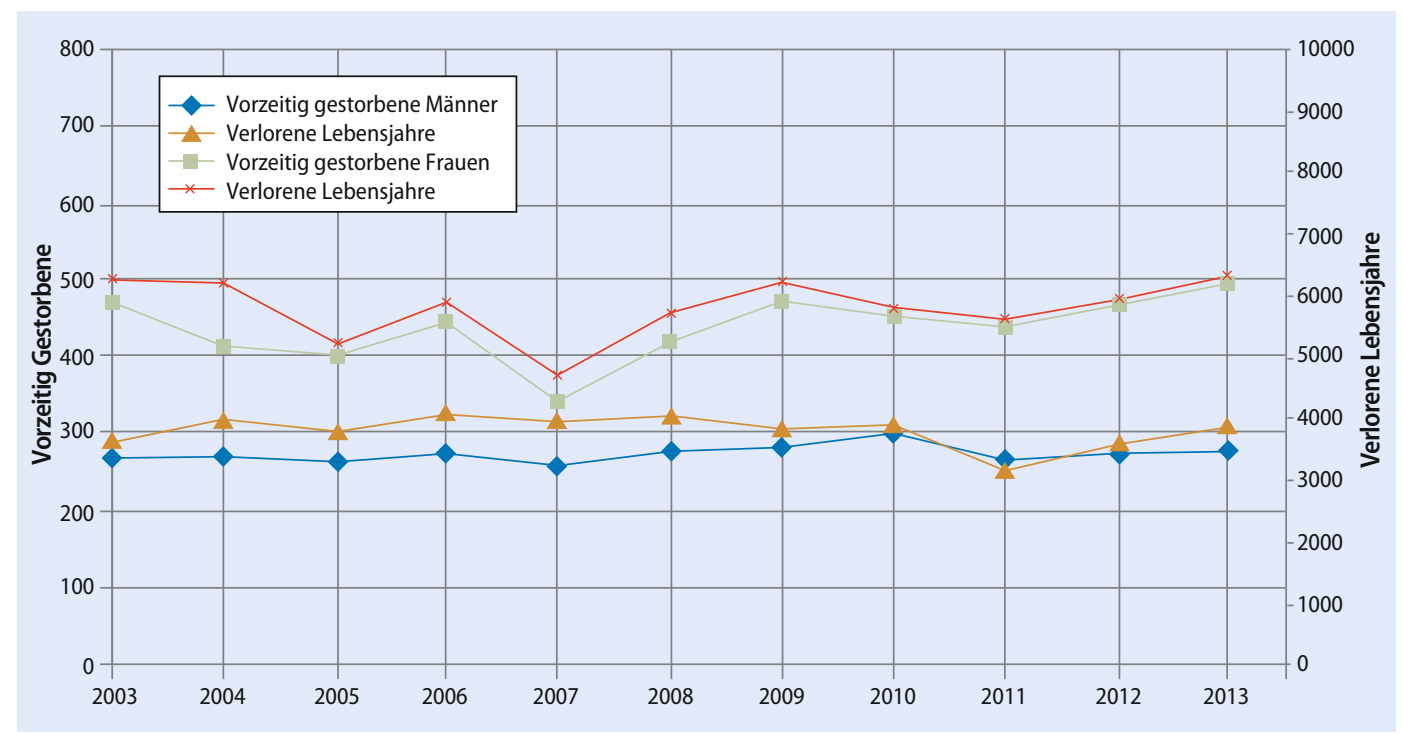

- Abb. 5.4 Veränderung der Anzahl vorzeitig Gestorbener und verlorener Lebensjahre aufgrund der MS (ICD-10: G.35) (2003 bis 2013)

Quelle: IGES - Gesundheitsberichterstattung des Bundes (2015b)

Gesellschaft (DMSG) in Nordrhein-Westfalen untersuchte die Lebensqualität der Erkrankten (Schipper et al. 2011). Zur Messung wurde der SF-36-Fragebogen eingesetzt, der die krankheitsübergreifende Lebensqualität auf Basis von acht Dimensionen erfasst. Dazu zählt neben Schmerzen u. a. auch die körperliche Rollenfunktion, die das Ausmaß der körperlichen Beeinträchtigung bei der Arbeit und anderen Aktivitäten erfasst. Die emotionale Rollenfunktion als weitere Dimension bildet ab, inwieweit emotionale Probleme die Arbeit oder andere Aktivitäten erschweren. Die Werte der einzelnen Skalen können zwischen 0 und 100 variieren, wobei hohe Werte auch für eine hohe Lebensqualität stehen. Die Ergebnisse der Patienten mit MS im Vergleich zur deutschen Normalbevölkerung sind in • Tab. 5.4 dargestellt.

Die Daten zeigen, dass an MS erkrankte Patienten alle Dimensionen der Lebensqualität niedriger bewerten als die deutsche Normalbevölkerung. Insbesondere in den Bereichen körperliche Funktionsfähigkeit sowie körperliche und emotionale Rollenfunktion ist die Lebensqualität geringer als bei Personen ohne MS. Auch an MS Erkrankte mit kurzer Krankheitsdauer ( $<2$ Jahren) und mit ge- ringer Behinderung erleben - mit Ausnahme der Dimension Schmerzen - in allen Bereichen eine signifikant verringerte Lebensqualität im Vergleich zur Normalbevölkerung. Einschränkend muss erwähnt werden, dass die Befragten im Vergleich zu Patienten im DMSG-Datensatz älter und schwerwiegender von der MS betroffen sind (Schipper et al. 2011).

Eine Befragung von 757 Mitgliedern des Sächsischen Landesverbandes der DMSG zeigt, dass bei $90 \%$ der Befragten die Erkrankung den Lebensalltag beeinflusst (Voigt et al. 2007). Etwa $80 \%$ der Teilnehmer berichten über Auswirkungen auf ihre Freizeitgestaltung. Zudem beeinflusst die Erkrankung Freundschaften (42\%), die Partnerschaft (37\%) und die Familienplanung (23\%). Mehr als $90 \%$ der Befragten berichten, dass sie bei der Verrichtung alltäglicher Tätigkeiten beispielsweise beim Säubern der Wohnung oder dem Zubereiten von Mahlzeiten unterstützt werden. Hilfe bekommen sie dabei überwiegend durch Familienmitglieder (Ehepartner: 77 \%, Kinder: 58 \%, Eltern: $35 \%$ ). Im Hinblick auf die Lebensqualität wirken sich neben dem Alter vor allem krankheitsspezifische Faktoren wie die Gehfähigkeit, Blasen- und Stuhl- 
- Tab. 5.4 Lebensqualität von Patienten mit MS im Vergleich zur Normalbevölkerung gemessen mittels SF-36 (IGES - Schipper et al. (2011))

\begin{tabular}{|l|l|l|}
\hline Skalen des SF-36 & Patienten mit MS & Normalbevölkerung \\
\hline Körperliche Funktionsfähigkeit & 44,34 & 85,93 \\
\hline Körperliche Rollenfunktion & 36,12 & 82,19 \\
\hline Schmerzen & 62,04 & 67,17 \\
\hline Allgemeine Gesundheitswahrnehmung & 44,95 & 66,76 \\
\hline Vitalität & 39,67 & 59,81 \\
\hline Soziale Funktionsfähigkeit & 63,76 & 85,95 \\
\hline Emotionale Rollenfunktion & 58,33 & 88,61 \\
\hline Psychisches Wohlbefinden & 61,28 & 72,07 \\
\hline 1 Für die Daten zur Normalbevölkerung wurden die Ergebnisse des Bundes-Gesundheitssurveys 1998 herangezogen.
\end{tabular}

probleme oder psychische Störungen negativ aus. Die sächsische Stichprobe ist durch ältere Patienten (Durchschnittsalter 50,5 Jahre) in fortgeschrittenem Krankheitsstadium gekennzeichnet (Voigt et al. 2007).

Patienten mit MS weisen auch im Vergleich mit Betroffenen anderer chronischer Krankheiten wie Rheumatoide Arthritis, Diabetes oder Epilepsie eine niedrigere Lebensqualität auf (Hermann et al. 1996; Rudick et al. 1992; Voigt et al. 2007).

Eine Möglichkeit, die intangiblen Kosten zu berechnen, besteht darin, den Verlust an Lebensqualität durch QALY (Quality adjusted life years) zu ermitteln. Ein QALY stellt den Nutzwert für ein Lebensjahr dar. Ein QALY von 1 bedeutet ein Jahr in vollständiger Gesundheit, während ein QALY von 0 dem Versterben entspricht (Schöffski u. Graf von der Schulenburg 2000). Die intangiblen Kosten können ermittelt werden, indem die QALY multipliziert werden mit der Zahlungsbereitschaft in der Gesellschaft, d. h. mit dem Geldbetrag, den die Gesellschaft bereit ist, für ein zusätzliches QALY zu zahlen. Die Krankheitskostenstudie von Kobelt et al. (2006) berechnet die intangiblen Kosten der MS in Deutschland. Der Verlust der Lebensqualität bei Patienten mit MS im Vergleich zur Gesamtbevölkerung wird dabei auf 0,2 QALY pro Jahr geschätzt. Bei einer angenommen Zahlungsbereitschaft von $50.000 €$ für ein QALY beziffern sich die intangiblen Kosten der MS auf durch- schnittlich etwa $10.000 €$ pro Patient (Kobelt et al. 2006).

\subsection{Krankheitslast}

Daten zur Krankheitslast (englisch: Burden of Disease) kombinieren epidemiologische Informationen zur Mortalität und Morbidität zu einer Maßeinheit und ermöglichen dadurch den Vergleich zwischen verschiedenen Erkrankungen (Field u. Gold 1998; Murray et al. 2002; Plass et al. 2014). Die World Health Organization (WHO) veröffentlicht auf ihrer Website aktuelle Daten zur globalen Krankheitslast auf Basis von DALY (Disability-Adjusted Life Years) mit dem Ziel, gesundheitspolitische Entscheidungen in den Regionen und Ländern zu unterstützen. DALY quantifizieren die Abweichung vom optimalen Gesundheitszustand (Plass et al. 2014). Der Indikator wird gebildet durch die Kombination von verlorenen Lebensjahren durch vorzeitiges Versterben (Years of Life Lost due to premature death, YLL) ${ }^{1}$ und die in suboptimaler Gesundheit bzw. mit Behinderung gelebten Lebens-

1 Zur Berechnung der YLL wird die Anzahl der Verstorbenen infolge einer Erkrankung (nach Alter und Geschlecht) mit einer Standard-Lebenserwartung für beide Geschlechter in Höhe von 92 Jahren bei Geburt multipliziert (WHO Department of Health Statistics and Information Systems 2013) 


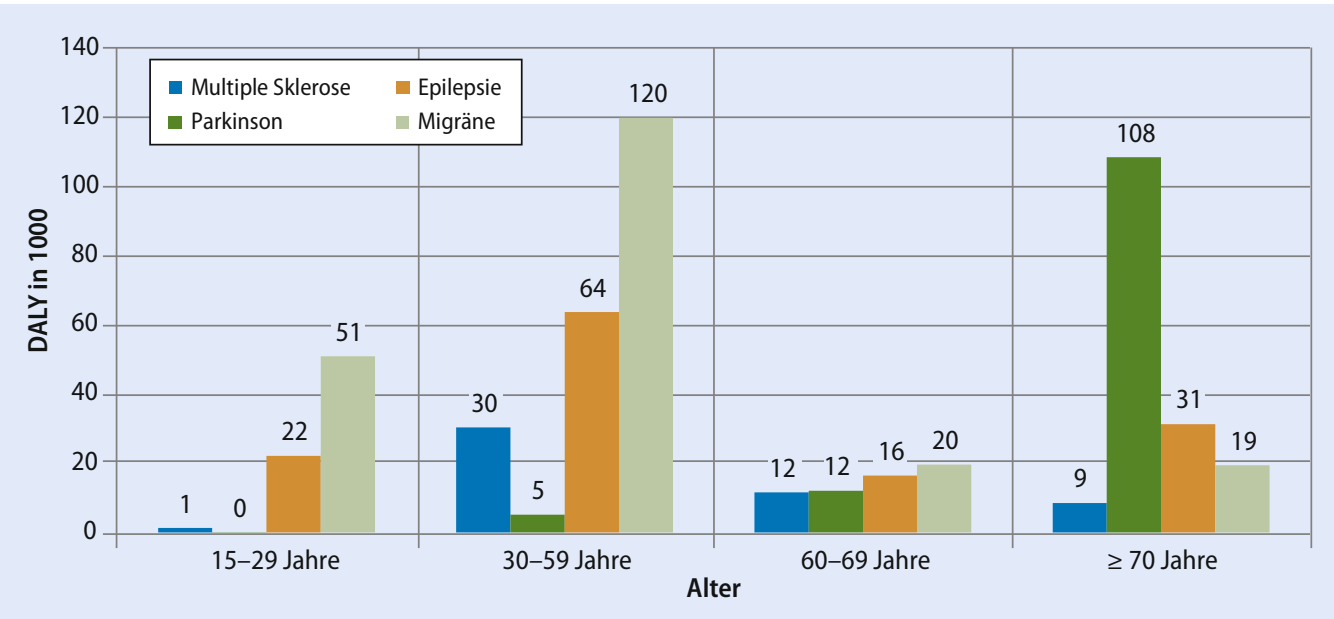

- Abb. 5.5 Vergleich der DALY zusammengesetzt aus verlorenen Lebensjahren durch vorzeitiges Versterben (YLL) und der Jahre mit Behinderung (YLD) für neurologische Erkrankungen in Deutschland für 2012 Quelle: IGES - WHO (2013)

jahre (Years Lived with Disability, YLD)² (WHO 2013).

MS verursacht insgesamt eine Krankheitslast von ca. 52.000 DALY, zusammengesetzt aus 35.000 verlorenen Lebensjahren durch vorzeitiges Versterben (YLL) und 17.000 Lebensjahren in suboptimaler Gesundheit (YLD). Die Krankheitslast der MS im Vergleich zu anderen neurologischen Erkrankungen in Abhängigkeit vom Alter ist in $\bullet$ Abb. 5.5 dargestellt. In der Altersgruppe der 30 - bis 59-Jährigen stellt die MS mit 30.000 DALY, neben der Migräne (120.000 DALY) und der Epilepsie (64.000 DALY), eine große Belastung dar. Dabei ist zu berücksichtigen, dass die Prävalenz von Migräne

2 Zur Berechnung der YLD wird die Prävalenz einer Erkrankung (nach Alter und Geschlecht) multipliziert mit einem krankheitsspezifischen Gewichtungsfaktor (»disability weights«). Der Gewichtungsfaktor bewertet die Auswirkungen der Erkrankung auf die Gesundheit auf einer Skala von 0 bis 1 . Ein Wert von 0 beschreibt einen optimalen Gesundheitszustand ohne Einschränkungen und der Wert 1 wird mit dem Tod gleichgesetzt. Die Faktoren wurden in populationsbasierten Studien ermittelt, in denen die Teilnehmer in paarweisen Vergleichen den präferierten Gesundheitszustand auswählen mussten (Plass et al. 2014). Die Gewichtungsfaktoren der MS liegen bei 0,198 Punkten für eine milde Verlaufsform, bei 0,445 Punkten für eine moderate Verlaufsform und bei 0,707 für eine schwere Verlaufsform (WHO Department of Health Statistics and Information Systems 2013). und Epilepsie höher ist als die von MS. Fast 60 \% der gesamten Krankheitslast der MS entstehen in dieser Alterskategorie. In den höheren Altersstufen sinkt die Krankheitslast. Mit ca. 12.000 DALY verursacht MS bei den 60- bis 69-Jährigen eine ähnlich große Krankheitslast wie Parkinson. Bei Personen ab 70 Jahren spielen die neurologischen Erkrankungen Parkinson (108.000 DALY) und Epilepsie (31.000 DALY) im Hinblick auf die Krankheitsbelastung eine größere Rolle als MS.

Zu berücksichtigen ist, dass die Prävalenz von Migräne und Epilepsie höher ist als die von MS und daher zu einer höheren Krankheitslast in den entsprechenden Altersgruppen führt.

Die Krankheitslast ist mit 33.000 DALY (64 \%) für Frauen mit MS deutlich höher als für Männer mit 19.000 DALY. Der Unterschied zwischen den Geschlechtern besteht in allen Altersgruppen. Die Anzahl der DALY für MS ist in den Jahren 2000 bis 2012 um $11 \%$ angestiegen. Die Zunahme ist vor allem auf einen Anstieg der YLL, insbesondere bei Frauen, zurückzuführen. 


\subsection{Finanzierung, Vergütung und Regularien der Arzneimittel- versorgung}

\subsubsection{Morbiditätsorientierter Risikostrukturausgleich}

Über den morbiditätsorientierten Risikostrukturausgleich wird die Höhe der Zuweisungen für die Krankenkasse zur Finanzierung ihrer Ausgaben aus dem Gesundheitsfond geregelt. Dazu erhält eine Krankenkasse zunächst für jeden Versicherten eine Grundpauschale in Höhe der durchschnittlichen Pro-Kopf-Ausgaben in der GKV (Bundesversicherungsamt 2008). Für das Jahr 2015 liegt diese Grundpauschale bei $2.797 €$ (Bundesversicherungsamt 2014). Um dem unterschiedlichen Versorgungsbedarf zwischen den Kassen gerecht zu werden, wird die Grundpauschale durch ein System von $\mathrm{Zu}$ - und Abschlägen ergänzt. Dabei wird neben den Versichertenmerkmalen Alter, Geschlecht und Bezug von Erwerbsminderungsrente auch die Krankheitslast auf Basis von 80 ausgewählten Krankheiten berücksichtigt. Die 80 selektierten Krankheiten stellen kostenintensive Erkrankungen dar sowie Krankheiten mit schwerwiegenden Verläufen, bei denen die durchschnittlichen Ausgaben pro Versicherten mindestens $50 \%$ höher sind als die durchschnittlichen Pro-Kopf-Leistungsausgaben für alle Versicherten (Bundesversicherungsamt 2008). Für gesunde Versicherte muss die Krankenkasse in der Regel, mit Ausnahme von Neugeborenen und sehr alten Menschen ( $\geq 90$ Jahre), in Abhängigkeit vom Alter und Geschlecht des Versicherten, einen Abschlag zahlen. Für Versicherte, die eine der 80 ausgewählten Erkrankungen haben, erhält die Krankenkasse einen Morbiditätszuschlag, der die durchschnittlichen durch die Krankheit verursachten Ausgaben widerspiegelt. Jede der 80 Krankheiten wird dazu hierarchisierten Morbiditätsgruppen (HMG) zugeordnet, für die ein entsprechender Zuschlag festgelegt wird. Für die HMG »Multiple Sklerose ohne Dauermedikation « liegt der vorläufige monatliche Zuschlag für 2015 bei $294,80 €$. Für die HMG »Multiple Sklerose mit Dauermedikation« liegt der Zuschlag bei 1.261,02 €.

\subsubsection{Vergütung stationärer Leistungen}

Stationäre Leistungen werden über Fallpauschalen (Diagnosis Related Groups, DRG) vergütet, die das leistungserbringende Krankenhaus mit dem Kostenträger abrechnet. Die gesetzliche Grundlage bildet $\$ 17 \mathrm{~b}$ des Krankenhausfinanzierungsgesetzes. Jedem individuellen Behandlungsfall wird auf Basis der Diagnose, der erbrachten Prozeduren, der Art der Behandlung und des Schweregrades der Erkrankung eine DRG-Fallpauschale zugeordnet. Zur Berechnung der Fallpauschale wird jeder DRG - in Abhängigkeit vom Behandlungsaufwand - ein Zahlenwert, die sogenannte Bewertungsrelation, zugeordnet. Diese wird dann multipliziert mit einem Basisfallwert, der den Basispreis für die einzelnen DRG-Leistungen darstellt (DIMDI 2015).

Die geschätzten Gesamtausgaben der MS im stationären Bereich auf Basis der DRG-Daten des Statistisches Bundesamtes liegen bei 136,4 Mio. € (GKV-Spitzenverband et al. 2012; InEK 2013; Statistisches Bundesamt 2013) (eigene Berechnung). In - Tab. 5.5 sind die im Jahr 2013 ausgabenintensivsten DRG (mindestens 0,5 Mio. $€$ ) für die Hauptdiagnose MS (ICD-10: G35) dargestellt. Fast $80 \%$ der Ausgaben (108,4 Mio. €) werden von der DRG B68D »Multiple Sklerose und zerebellare Ataxie (ein Belegungstag oder ohne äußerst schwere Komplikationen oder Komorbiditäten, Alter $>15$ Jahre, ohne komplexe Diagnose) « verursacht. Für die Berechnung der Ausgaben je DRG wurde die Bewertungsrelation der einzelnen DRGs mit dem Bundesbasisfallwert in Höhe von 3.068,37€ für das Jahr 2013 multipliziert. Der Bundesbasisfallwert wird zwischen der Deutschen Krankenhausgesellschaft (DKG), dem GKV-Spitzenverband und dem Verband der privaten Krankenversicherung (PKV) jedes Jahr vertraglich vereinbart und gibt die Grenzwerte für die Landesbasisfallwerte vor (GKVSpitzenverband et al. 2012). Die stationären Ausgaben der MS sind wahrscheinlich unterschätzt, da krankenhausindividuell vereinbarte DRGs sowie Zusatzentgelte wie beispielsweise für Arzneimittel nicht berücksichtigt werden. 
Tab. 5.5 Die kostenintensivsten DRG ( $\geq 0,5$ Mio. €) für die Hauptdiagnose MS (ICD-10: G35) im Jahr 2013 (IGES Berechnung basierend auf Statistisches Bundesamt 2013 (GKV-Spitzenverband et al. 2012; InEK 2013)

\begin{tabular}{|c|c|c|c|c|}
\hline DRG & DRG-Bezeichnung & $\begin{array}{l}\text { Anzahl } \\
\text { der Fälle }\end{array}$ & $\begin{array}{l}\text { Bewertungs- } \\
\text { relation }\end{array}$ & $\begin{array}{l}\text { Ausgaben } \\
\text { in Mio. } €\end{array}$ \\
\hline B68D & $\begin{array}{l}\text { Multiple Sklerose und zerebellare Ataxie, ein Belegungstag oder } \\
\text { ohne äußerst schwere Komplikationen oder Komorbiditäten, } \\
\text { Alter > } 15 \text { Jahre, ohne komplexe Diagnose }\end{array}$ & 47.936 & 0,737 & 108,4 \\
\hline B48Z & $\begin{array}{l}\text { Frührehabilitation bei Multipler Sklerose und zerebellarer } \\
\text { Ataxie, nicht akuter Para- / Tetraplegie oder anderen neurolo- } \\
\text { gischen Erkrankungen }\end{array}$ & 3.024 & 1,850 & 17,2 \\
\hline $\mathrm{B} 68 \mathrm{~A}$ & $\begin{array}{l}\text { Multiple Sklerose und zerebellare Ataxie mit äußerst schweren } \\
\text { Komplikationen oder Komorbiditäten, mehr als ein Belegungstag }\end{array}$ & 1.013 & 1,773 & 5,5 \\
\hline $\mathrm{B} 68 \mathrm{~B}$ & $\begin{array}{l}\text { Multiple Sklerose und zerebellare Ataxie, ein Belegungstag oder } \\
\text { ohne äußerst schwere Komplikationen oder Komorbiditäten, } \\
\text { Alter < } 16 \text { Jahre }\end{array}$ & 348 & 0,979 & 1,0 \\
\hline B42B & $\begin{array}{l}\text { Frührehabilitation bei Krankheiten und Störungen des Nerven- } \\
\text { systems bis } 27 \text { Tage ohne neurologische Komplexbehandlung } \\
\text { des akuten Schlaganfalls }\end{array}$ & 46 & 3,641 & 0,5 \\
\hline B44B & $\begin{array}{l}\text { Geriatrische frührehabilitative Komplexbehandlung bei Krank- } \\
\text { heiten und Störungen des Nervensystems mit schwerer moto- } \\
\text { rischer Funktionseinschränkung, ohne neurologische Komplex- } \\
\text { behandlung des akuten Schlaganfalls }\end{array}$ & 67 & 2,434 & 0,5 \\
\hline
\end{tabular}

\subsubsection{Vergütung ambulanter Leistungen}

Die ambulante Versorgung der MS erfolgt in der Regel durch Neurologen, spezialisierte (Neuro)Radiologen sowie durch Hausärzte ( $\triangleright$ Kap. 6). Die Vergütung der vertragsärztlichen Leistungen erfolgt basierend auf dem Einheitlichen Bewertungsmaßstab (EBM). Dieser bestimmt den Inhalt der abrechnungsfähigen Leistungen und weist einer festgelegten Leistung durch die Vergabe von Punkten einen Wert $\mathrm{zu}^{3}$. Der bundesweit geltende EBM wird im Bewertungsausschuss zwischen Vertretern der Kassenärztlichen Bundesvereinigung und dem GKVSpitzenverbänden vereinbart. Die Abrechnung der Leistungen erfolgt pauschal über die Kassenärztlichen Vereinigungen. In Abhängigkeit der erbrachten Leistungen variiert die Vergütung für die Behandlung eines Patienten.

Ein Großteil der Therapie des akuten Schubes könnte ambulant erfolgen. In der ambulanten Ver- sorgung sind aber die vorgesehenen Strukturen nicht ausreichend vorhanden. Aus diesem Grund werden viele Patienten mit einem akuten Schub unnötigerweise sehr viel kostenintensiver stationär versorgt.

\subsubsection{Regulatorische Aspekte der Arzneimittelversorgung}

Am 1. Januar 2011 trat das Gesetz zur Neuordnung des Arzneimittelmarktes (AMNOG) in Kraft, wonach der Gemeinsame Bundesausschuss (G-BA) für die Bewertung des Nutzens von Arzneimitteln mit neuen Wirkstoffen zuständig ist ${ }^{4}(\triangleright$ Kap. 6). Die pharmazeutischen Unternehmen sind dazu verpflichtet, spätestens bei Markteintritt bzw. vier Wochen nach Zulassung eines neuen Anwendungsgebietes dem G-BA ein Dossier vorzulegen, in dem sie anhand der Zulassungsunterlagen und den klinischen Studien den Zusatznutzen des Arzneimit- 
tels im Vergleich zur vorher festgelegten zweckmäßigen Vergleichstherapie nachweisen. Zur Nutzenbewertung kann der G-BA das Institut für Qualität und Wirtschaftlichkeit im Gesundheitswesen (IQWiG) oder Dritte beauftragen ${ }^{5}$. Das IQWiG leitet dem G-BA seine Ergebnisse als Empfehlungen $\mathrm{zu}$, diese sind allerdings nicht bindend. Die Ergebnisse der Nutzenbewertung werden in Form von Beschlüssen veröffentlicht. Darin werden der $\mathrm{Zu}$ satznutzen des Arzneimittels gegenüber der zweckmäßigen Vergleichstherapie, das Ausmaß des Zusatznutzens, die zur Behandlung in Frage kommenden Patientengruppen, Anforderungen an eine qualitätsgesicherte Anwendung sowie die Therapiekosten festgelegt (Gemeinsamer Bundesausschuss 2015a).

Das Ergebnis der Nutzenbewertung bildet die Entscheidungsgrundlage dafür, welchen Erstattungspreis die gesetzlichen Krankenkassen bereit sind, für das neue Arzneimittel zu zahlen (Gemeinsamer Bundesausschuss 2015a). Wird einem Arzneimittel durch den G-BA ein Zusatznutzen zugewiesen, beginnen Preisverhandlungen zwischen dem GKV-Spitzenverband und dem pharmazeutischen Hersteller. Kommt es zu keiner Einigung, wird der Erstattungsbetrag von einer Schiedskommission festgelegt. Für neue oder in einem neuen Anwendungsgebiet zugelassene Medikamente ohne Zusatznutzen kann ein entsprechender Festbetrag festgelegt werden ${ }^{6}$.

Die methodischen Verfahren zur Nutzenbewertung können den interindividuell unterschiedlichen Krankheitsverläufen der MS nicht gerecht werden. Eine mittelwertbasierte Herangehensweise in der Bewertung eines Zusatznutzens eines sehr heterogenen Krankheitsbildes wie der MS vernachlässigt eine mitunter nicht geringe Anzahl an Patienten, die dennoch von einzelnen Therapien profitieren können.

In der Indikation MS waren bisher insgesamt vier neue Wirkstoffe (Fingolimod, Fampridin, Teriflunomid, Dimethylfumarat) Gegenstand der Nutzenbewertung. Die Ergebnisse der einzelnen Nutzenbewertungsverfahren für die jeweiligen Anwendungsgebiete der Arzneimittel sowie die zweckmäßige Vergleichstherapie sind in -Tab. 5.6 dargestellt.

$5 \S 35 a \mathrm{SGB} V$

$6 \S 35 a \mathrm{SGB} V$
Der Wirkstoff Fingolimod ist seit 2011 zur Behandlung der hochaktiven RRMS bei Patienten, die nicht auf einen vollständigen und angemessenen Zyklus einer Interferon- $ß$-Therapie angesprochen haben und bei Patienten mit rasch fortschreitender schwerer RRMS zugelassen sowie seit 2014 für Patienten mit hochaktiver RRMS, die mit einer anderen Therapie als Interferon- $\beta$ vorbehandelt wurden (Gemeinsamer Bundesausschuss 2014a). Der G-BA kommt in seiner Nutzenbewertung vom Oktober 2015 zur Einschätzung, dass bei Patienten mit hochaktiver, noch nicht vollständig mit Interferon- $ß$ behandelter RRMS ein Hinweis auf einen beträchtlichen Zusatznutzen und bei Patienten mit rasch fortschreitender schwerer RRMS ein geringer $\mathrm{Zu}$ satznutzen vorliegt (Gemeinsamer Bundesausschuss 2015b). Für Patienten mit hochaktiver RRMS, die bereits eine vollständige Vorbehandlung mit Beta-Interferonen (IFN- $B$ 1a) erhalten haben, war im Vergleich zu Glatirameracetat der Zusatznutzen nicht belegt.

Fampridin ist zur Verbesserung der Gehfähigkeit von Erwachsenen mit MS mit Gehbehinderung (Grad 4-7 auf der EDSS-Behinderungsskala) zugelassen. Die vom G-BA festgelegte zweckmäßige Vergleichstherapie war Krankengymnastik. Aus Sicht des G-BA konnte der Zusatznutzen von Fampridin als zusätzliche Therapie zur Krankengymnastik im Vergleich zur Krankengymnastik allein nicht nachgewiesen werden (Gemeinsamer Bundesausschuss 2012a).

Teriflumomid ist seit 2013 zur Behandlung der RRMS zugelassen. Als zweckmäßige Vergleichstherapie bestimmte der G-BA Interferon- $\beta$ (1a oder 1b) oder Glatirameracetat. Studienergebnisse zeigten u.a. keine relevanten Unterschiede zwischen den beiden Präparaten in Hinblick auf die Morbidität und Lebensqualität. Der G-BA entschied daher, dass der Zusatznutzen von Teriflunomid nicht belegt ist (Gemeinsamer Bundesausschuss 2014b).

Dimethylfumarat ist seit 2014 zur Behandlung der RRMS zugelassen. Als zweckmäßige Vergleichstherapie bestimmte der G-BA Interferon- $\beta$ (1a oder 1b) oder Glatirameracetat. Der G-BA entschied, dass der Zusatznutzen von Dimethylfumarat nicht belegt ist. Er begründet diese Entscheidung damit, dass trotz vorhandener Studiendaten kein direkter 
Tab. 5.6 Ergebnisse bisheriger Nutzenbewertungsverfahren für zur Therapie von MS empfohlener Wirkstoffe (IGES - Gemeinsamer Bundesausschuss (2015b; 2014a; 2014b; 2014c; 2012a)

\begin{tabular}{|c|c|c|c|}
\hline Wirkstoff & Anwendungsgebiet & $\begin{array}{l}\text { Zweckmäßige Vergleichs- } \\
\text { therapie }\end{array}$ & Zusatznutzen G-BA \\
\hline \multirow[t]{5}{*}{ Fingolimod } & $\begin{array}{l}\text { Patienten mit hochaktiver RRMS mit } \\
\text { hoher Krankheitsaktivität ohne ausrei- } \\
\text { chende krankheitsmodifizierende } \\
\text { Therapie mit IFN- } \beta\end{array}$ & $\begin{array}{l}\text { Fortführung der mit IFN- } \beta \text { be- } \\
\text { gonnenen krankheitsmodifizie- } \\
\text { renden Therapie mit einer ge- } \\
\text { mäß Zulassung optimierten } \\
\text { Dosierung bis zu einem ange- } \\
\text { messenen Zyklus }\end{array}$ & beträchtlich \\
\hline & Rasch fortschreitende schwere RRMS & $\begin{array}{l}\text { Glatirameracetat oder IFN- } \beta \\
\text { (1a oder } 1 b)\end{array}$ & gering \\
\hline & $\begin{array}{l}\text { Patienten mit hochaktiver RRMS mit } \\
\text { hoher Krankheitsaktivität die nicht auf } \\
\text { einen vollständigen und angemesse- } \\
\text { nen Zyklus mit IFN- } \beta \text { angesprochen } \\
\text { haben }\end{array}$ & Glatirameracetat & nicht belegt \\
\hline & $\begin{array}{l}\text { Patienten mit hochaktiver RRMS } \\
\text { mit hoher Krankheitsaktivität ohne } \\
\text { ausreichende krankheitsmodifizierende } \\
\text { Therapie (anders als INF- } \beta \text { ) }\end{array}$ & $\begin{array}{l}\text { Fortführung der mit Glatiramer- } \\
\text { acetat oder INF- } \beta \text { begonnenen } \\
\text { krankheitsmodifizierenden } \\
\text { Therapie mit einer gemäß Zulas- } \\
\text { sung optimierten Dosierung bis } \\
\text { zu einem angemessenen Zyklus. } \\
\text { Ist die krankheitsmodifizierende } \\
\text { Therapie mit anderen Arzneimit- } \\
\text { teln begonnen worden, ist ein } \\
\text { Wechsel auf Glatirameracetat } \\
\text { oder INF- } \beta \text { mit einer gemäß } \\
\text { Zulassung optimierten Dosie- } \\
\text { rung bis zu einem angemes- } \\
\text { senen Zyklus durchzuführen }\end{array}$ & nicht belegt \\
\hline & $\begin{array}{l}\text { Patienten mit hochaktiver RRMS mit } \\
\text { hoher Krankheitsaktivität, die nicht auf } \\
\text { einen vollständigen und angemesse- } \\
\text { nen Zyklus mit mindestens einer krank- } \\
\text { heitsmodifizierenden Therapie (anders } \\
\text { als INF- } \beta \text { ) angesprochen haben }\end{array}$ & $\begin{array}{l}\text { Glatirameracetat oder INF- } \beta \\
\text { (1a oder } 1 b)\end{array}$ & nicht belegt \\
\hline Fampridin & $\begin{array}{l}\text { Verbesserung der Gehfähigkeit von } \\
\text { erwachsenen Patienten mit MS } \\
\text { (EDSS } 4 \text { bis } 7 \text { ) }\end{array}$ & $\begin{array}{l}\text { Krankengymnastik nach Heilmit- } \\
\text { telrichtlinie und optimierte } \\
\text { MS-Standardtherapie }\end{array}$ & nicht belegt \\
\hline Teriflunomid & $\begin{array}{l}\text { Behandlung erwachsener Patienten } \\
\text { mit RRMS }\end{array}$ & $\begin{array}{l}\text { Glatirameracetat oder IFN- } \beta \text { ( } 1 \text { a } \\
\text { oder } 1 b \text { ) }\end{array}$ & nicht belegt \\
\hline Dimethylfumarat & $\begin{array}{l}\text { Behandlung erwachsener Patienten } \\
\text { mit RRMS }\end{array}$ & $\begin{array}{l}\text { Glatirameracetat oder IFN- } \beta \text { (1a } \\
\text { oder } 1 \mathrm{~b} \text { ) }\end{array}$ & nicht belegt \\
\hline
\end{tabular}


Vergleich zwischen dem Arzneimittel und der Vergleichstherapie durchgeführt wurde (Gemeinsamer Bundesausschuss 2014c).

Die Wirkstoffe Natalizumab und Alemtuzumab durchliefen das Nutzenbewertungsverfahren nach
$\$ 35$ a SGB V nicht. Natalizumab wurde bereits im Jahr 2006 - vor Beginn des Nutzenbewertungsverfahrens - eingeführt und auch Alemtuzumab war bereits zuvor für die Behandlung von B-Zell-Leukämien zugelassen.

Open Access Dieses Kapitel wird unter der Creative Commons Namensnennung-Nicht kommerziell 4.0 International Lizenz (http://creativecommons.org/licenses/by-nc/4.0/deed.de) veröffentlicht, welche für nicht kommerzielle Zwecke die Nutzung, Vervielfältigung, Bearbeitung, Verbreitung und Wiedergabe in jeglichem Medium und Format erlaubt, sofern Sie den/die ursprünglichen Autor(en) und die Quelle ordnungsgemäß nennen, ein Link zur Creative Commons Lizenz beifügen und angeben, ob Änderungen vorgenommen wurden.

Etwaige Abbildungen oder sonstiges Drittmaterial unterliegen ebenfalls der genannten Creative Commons Lizenz, sofern sich aus der Abbildungslegende oder der Quellreferenz nichts anderes ergibt. Sofern solches Drittmaterial nicht unter der genannten Creative Commons Lizenz steht, ist eine Vervielfältigung, Bearbeitung oder öffentliche Wiedergabe nur mit vorheriger Zustimmung des betreffenden Rechteinhabers oder auf der Grundlage einschlägiger gesetzlicher Erlaubnisvorschriften zulässig.

\section{Literatur}

Bundesversicherungsamt (2014) Bekantmachung zum Gesundheitsfond Nr. 1/2015.

Bundesversicherungsamt (2008) So funktioniert der neue Risikostrukturausgleich im Gesundheitsfond. http://www. bundesversicherungsamt.de/fileadmin/redaktion/ Risikostrukturausgleich/Wie_funktioniert_Morbi_RSA.pdf [Abruf am: 20.11.2015].

DIMDI (2015) G-DRG-System - Fallpauschalen in der stationären Versorgung. Deutsches Institut für Medizinische Dokumentation und Information. https://www.dimdi.de/ static/de/klassi/icd-10-gm/anwendung/zweck/g-drg/ [Abruf am: 28. Mai 2015].

Evers SM, Struijs JN, Ament AJ, van Genugten ML, Jager JH, van den Bos GA (2004) International comparison of stroke cost studies. Stroke 35(5), 1209-1215. DOI: 10.1161/01.STR.0000125860.48180.48.

Field MJ, Gold MR (1998) Summarizing Population Health. Directions for the Development and Application of Population Metrics. Washington: National Academy Press.

Flachenecker P, Stuke K, Elias W, Freidel M, Haas J, PitschnauMichel D, Schimrigk S, Zettl UK, Rieckmann P (2008) Multiple sclerosis registry in Germany: results of the extension phase 2005/2006. Deutsches Ärzteblatt 105(7), 113-119. DOI: 10.3238/arztebl.2008.0113.

Gemeinsamer Bundesausschuss (2012a) Beschluss des Gemeinsamen Bundesausschusses über eine Änderung der Arzneimittel-Richtlinie (AM-RL) Anlage XII - Beschlüsse über die Nutzenbewertung von Arzneimitteln mit neuen Wirkstoffen nach $\S 35$ a SGB V - Fampridin vom 2. August 2012. Berlin: Gemeinsamer Bundesausschuss.
Gemeinsamer Bundesausschuss (2014a) Beschluss des Gemeinsamen Bundesausschusses über eine Änderung der Arzneimittel-Richtlinie (AM-RL): Anlage XII - Beschlüsse über die Nutzenbewertung von Arzneimitteln mit neuen Wirkstoffen nach § 35a SGB V - Fingolimod (neues Anwendungsgebiet) vom 18. Dezember 2014. Berlin: Gemeinsamer Bundesausschuss.

Gemeinsamer Bundesausschuss (2015a) Die Nutzenbewertung von Arzneimitteln gemäß § 35a SGB V. https:// www.g-ba.de/institution/themenschwerpunkte/arzneimittel/nutzenbewertung35a/[Abruf am: 11. Juni 2015].

Gemeinsamer Bundesausschuss (2015b) Beschluss des Gemeinsamen Bundesausschusses über eine Änderung der Arzneimittel-Richtlinie (AM-RL): Anlage XII - Beschlüsse über die Nutzenbewertung von Arzneimitteln mit neuen Wirkstoffen nach § 35a SGB V - Fingolimod (Ablauf der Befristung) vom 1. Oktober 2015. Berlin: Gemeinsamer Bundesausschuss.

Gemeinsamer Bundesausschuss (2014b) Tragende Gründe zum Beschluss des Gemeinsamen Bundesausschusses über eine Änderung der Arzneimittel-Richtlinie (AM-RL): Anlage XII - Beschlüsse über die Nutzenbewertung von Arzneimitteln mit neuen Wirkstoffen nach § 35a SGB V Teriflunomid vom 20. März 2014. Berlin: Gemeinsamer Bundesausschuss.

Gemeinsamer Bundesausschuss (2014c) Beschluss des Gemeinsamen Bundesausschusses über eine Änderung der Arzneimittel-Richtlinie (AM-RL): Anlage XII - Beschlüsse über die Nutzenbewertung von Arzneimitteln mit neuen Wirkstoffen nach § 35a SGB V - Dimethylfumarat vom 16. Oktober 2014. Berlin: Gemeinsamer Bundesausschuss. 
Gesundheitsberichterstattung des Bundes (2015a) Intangible Kosten. Bonn: Statistisches Bundesamt. www.gbe-bund. de/gbe10/abrechnung.prc_abr_test_logon?p_uid= gast $\&$ p_aid=0\&p_knoten=FID\&p_sprache=D\&p_ suchstring=9102 [Abruf am: 28. Mai 2015].

Gesundheitsberichterstattung des Bundes (2015b) Vorzeitige Sterblichkeit (Anzahl, je 100.000 Einwohner, verlorene Lebensjahre - mit/ohne Altersstandardisierung, Tod unter 65/70 Jahren - ab 1998). Gliederungsmerkmale: Jahre, Region, Geschlecht, ICD-10, Art der Standardisierung. Bonn: Statistisches Bundesamt. https://www. gbe-bund.de/oowa921-install/servlet/oowa/aw92/ dboowasys921.xwdevkit/xwd_init?gbe.isgbetol/xs_ start_neu/\&p_aid=3\&p_aid=32960037\&nummer= 562\&p_sprache $=D \& p \_i n d s p=11220874 \& p \_a i d=$ 32440284 [Abruf am: 11. Juni 2015].

GKV-Spitzenverband, Berlin, Verband der privaten Krankenversicherung e.V., Köln, Deutsche Krankenhausgesellschaft, Berlin (2012) Vereinbarung gemäß § 10 Abs. 9 KHEntgG für den Vereinbarungszeitraum 2013 vom 17.12.2012.

Graf von der Schulenburg JM, Greiner W (2007) Gesundheitsökonomik. 2. Auflage. Tübingen: Mohr Siebeck. ISBN: 978-3-16-149060-6.

Hapfelmeier A, Dippel FW, Schinzel S, Holz B, Seiffert A, Mäurer M (2013) Aktuelle Aspekte zur Versorgungssituation und zu den Behandlungskosten bei Patienten mit Multipler Sklerose in Deutschland. Gesundheitsökonomie \& Qualitätsmanagement 18, 210-216. DOI: 10.1055/ s-0033-1335883.

Hermann BP, Vickrey B, Hays RD, Cramer J, Devinsky O, Meador K, Perrine K, Myers LW, Ellison GW (1996) A comparison of health-related quality of life in patients with epilepsy, diabetes and multiple sclerosis. Epilepsy Research 25(2), 113-118. ISSN: 0920-1211.

IGES Institut (2014) Neurologische und psychiatrische Versorgung aus sektorenübergreifender Perspektive. Studie im Auftrag von Berufsverband Deutscher Nervenärzte e.V. (BVDN), Berufsverband Deutscher Neurologen e.V. (BDN), Berufsverband Deutscher Psychiater e.V (BVDP), Kassenärztliche Bundesvereinigung (KBV) / Zentralinstitut für die kassenärztliche Versorgung in Deutschland (ZI), Deutsche Gesellschaft für Neurologie e.V (DGN). Ergebnisbericht. Berlin: IGES Institut.

InEK (2013) Fallpauschalen-Katalog. G-DRG-Version 2013. Institut für das Entgeldsystem im Krankenhaus. http:// www.g-drg.de/cms/content/download/3876/31661/ version/4/file/Anlagen_DRG-Entgeltkatalog_2013_ 20121019_20121023.xls [Abruf am: 11. Juni 2015].

IQWiG (2009) Arbeitspapier Kostenbestimmung. Version 1.0 vom 12.10.2009. KöIn: Institut für Qualität und Wirtschaftlichkeit im Gesundheitswesen (IQWiG).

Karampampa K, Gustavsson A, Miltenburger C, Eckert B (2012a) Treatment experience, burden and unmet needs (TRIBUNE) in MS study: results from five European countries. Multiple Sclerosis 18(2 Suppl), 7-15. DOI: $10.1177 / 1352458512441566$.
Karampampa K, Gustavsson A, Miltenburger C, Neidhardt K, Lang M (2012b) Treatment experience, burden and unmet needs (TRIBUNE) in MS study: results from Germany. Multiple Sclerosis 18(2 Suppl), 23-27. DOI: $10.1177 / 1352458512441566 b$.

Kern S, Kühn M, Ziemssen T (2013) Chronisch krank und ohne Arbeit? Eine aktuelle Analyse zur Erwerbstätigkeit bei Multipler Sklerose. Fortschritte der Neurologie und Psychiatrie 81, 95-103. DOI: 10.1055/s-00321330286.

Kobelt G, Berg J, Lindgren P, Elias WG, Flachenecker P, Freidel M, Konig N, Limmroth V, Straube E (2006) Costs and quality of life of multiple sclerosis in Germany. The European journal of health economics 7 Suppl 2, S34-44. DOI: 10.1007/s10198-006-0384-8.

Löwel H, Hörmann A, Döring A, Heier M, Meisinger C, Schneider A, Kaup U, Gösele U, Hymer H (2006) Koronare Herzkrankheit und akuter Myokardinfarkt. Heft 33. Gesundheitsberichterstattung des Bundes. Berlin: Robert Koch-Institut.

msfp (2014) Aktuelles aus dem MS-Register der DMSG, Bundesverband e.V. Hannover: MS Forschungs- und Projektentwicklungs-gGmbH.

Murray CJL, Salomon JA, Mathers CD, Lopez AD (2002) Summary Measures of Population Health. Concepts, Ethics, Measurement and Applications. Geneva: World Health Organization. ISBN: 9241545518.

Plass D, Vos T, Hornberg C, Scheidt-Nave C, Zeeb H, Krämer A (2014) Entwicklung der Krankheitslast in Deutschland. Ergebnisse, Potenziale und Grenzen der Global Burden of Disease-Studie. Deutsches Ärzteblatt 111(38), 629-638. DOI: 10.3238/arztebl.2014.0629.

Reese JP, John A, Wienemann G, Wellek A, Sommer N, Tackenberg B, Balzer-Geldsetzer M, Dodel R (2011) Economic burden in a German cohort of patients with multiple sclerosis. European Neurology 66(6), 311-321. DOI: 10.1159/000331043.

Rudick RA, Miller D, Clough JD, Gragg LA, Farmer RG (1992) Quality of life in multiple sclerosis. Comparison with inflammatory bowel disease and rheumatoid arthritis. Archives of Neurology and Psychiatry 49(12), 1237-1242. ISSN: 0003-9942.

Schipper S, Wiesmeth S, Wirtz M, Twork S, Kugler J (2011) Krankheitsverarbeitungsstile und gesundheitsbezogene Lebensqualität bei Multiple-Sklerose-Erkrankten. Psychotherapie, Psychosomatik, Medizinische Psychologie 61(8), 347-355. DOI: 10.1055/s-0031-1275744.

Schöffski O, Graf von der Schulenburg JM (2000) Gesundheitsökonomische Evaluationen. Heidelberg: Springer. ISBN: 9783642216992.

Statistisches Bundesamt (2013) Gesundheit. Fallpauschalenbezogene Krankenhausstatistik (DRG-Statistik) Diagnosen, Prozeduren, Fallpauschalen und Case Mix der vollstationären Patientinnen und Patienten in Krankenhäusern. Fachserie 12 Reihe 6.4. Wiesbaden: Statistisches Bundesamt. 
Statistisches Bundesamt (2010) Gesundheit. Krankheitskosten 2002, 2004, 2006 und 2008. Wiesbaden: Statistisches Bundesamt.

Voigt K, Worm I, Klewer J, Twork S, Kugler J, German Society for Multiple S (2007) Lebens- und Versorgungsqualität von Mitgliedern des Sächsischen Landesverbandes der Deutschen Mutiple Sklerose Gesellschaft. Gesundheitswesen 69(8-9), 457-463. DOI: 10.1055/s-2007-985866.

WHO Department of Health Statistics and Information Systems (2013) WHO methods and data sources for global burden of disease estimates 2000-2011. Global Health Estimates Technical Paper WHO/HIS/HSI/GHE/2013.4. Genf: World Health Organization. 